\title{
What Is Life: An Informational Model of the Living Structures
}

\author{
Florin Gaiseanu ${ }^{1,2}$ \\ ${ }^{1}$ Science and Technology of Information/Microsystems, IMT (Institute of Microtechnology), Bucharest, Romania \\ ${ }^{2}$ Science and Technology of Information/Microtechnology, CNM (Centro Nacional de Microelectrónica), Barcelona, Spain
}

\section{Email address:}

fgtext@yahoo.es

\section{To cite this article:}

Florin Gaiseanu. What Is Life: An Informational Model of the Living Structures. Biochemistry and Molecular Biology.

Vol. 5, No. 2, 2020, pp. 18-28. doi: 10.11648/j.bmb.20200502.12

Received: October 5, 2020; Accepted: October 17, 2020; Published: October 26, 2020

\begin{abstract}
Schröedinger's question "what is life?" was a real challenge for the scientific community and this still remains as an opened question, because in spite of the important advances in various scientific branches like philosophy, biology, chemistry and physics, each of them assesses life from its particular point of view to explain the life' characteristic features, so not a coherent and well structured general model of life was reported. In this paper life is approached from informational perspective, starting from earlier Draganeacu's philosophic concepts, showing that actually life is structured by matter and information. Therefore, it is analyzed the carbon-matter and its properties on the basis of which the living structures are composed, giving rise not only of a considerable number of carbon-based compounds, but serving now beside silicon, as an useful material for micro/nanostructure applications. Such specific properties refers to the high ability of carbon to associate/dissociate in chemical reactions regulated/facilitated by informational (Bit unit) YES/NO bistable mechanisms to form macro/small molecules with complementary properties, reactive info-functional pathways of transduction, relaying, amplification, integration, spreading, modulation, activation and positive/negative feedback reactions, like in the informational microelectronic/microsystems circuits. It is argued that the negentropy invoked earlier in Schrödinger's analysis is a consequence of informational-assisted structuration/organization of the cell and human organism. From the analysis of inter/intra-communication mechanisms in the cell and comparing with the outcomes described by the Informational Model of Human Body, it is deduced that the living organisms operate on the basis of three main streaming circuits assuring the living functions: (1) the metabolic matter-related circuit; (2) the operative informational circuit; (3) the epigenetic informational circuit for the gradual integration of information in the central informational structure - DNA. It is founded on these bases the Informational Model of the Living Structures, and the Informational System of the Living Structures (ISLS), with similar functions on the entire living scale size, from unitary to multicellular living structures, composed by seven informational systems, namely [CASI (center of acquisition and storing of information), CDC (center of decision and command), IRSS (info-reactive sentient system), MIS (maintenance info-system), GTS (genetic transmission system), IGG (info-genetic generator) and IC (info-connection)] $]_{\text {ISLS, }}$ and are identified the specific functions of each of them. The living structures operate thus like self-"polarized" bipolar info-matter informational devices by means of the stand-by metabolic matter-related circuit, and react/respond to the external/internal informational stimuli, which modulate their functionality, returning an external reaction signal ("attitude"), for adaptation and survival.
\end{abstract}

Keywords: Life/Information, Entropy/Negentropy, Living/Non-living Matter Structuration, Cell/Human Operability, Self-ordering/Info-organization, Informational Model of Living Structures

\section{Introduction}

Since immemorial times, the human asked himself "What is life?" [1], a question which remained still actual, after Schrödinger, the famous physicist in quantum mechanics field, launched this question explicitly with more than seven decades ago, as a challenge to the scientific community to find an adequate response. In our informational era, we are still asking ourselves this question, especially when we have to respond to our deep problems of our life in present and future, requiring a difficult decision [2], when we have to reconsider our health equilibrium [3], or when the age or 
unexpected situations advances inexorably to new unknown horizons [4]. Therefore, this question is so important for all of us, and the scientific community still tries to find an adequate response to this complex problem, from the perspective of various scientific branches, starting from philosophy concerning the mind-body problem and consciousness [1] to physics at small cell scale $[5,6]$ and at high scale [7], debating especially the thermodynamics properties of living, passing through biology and chemistry [8] relating the structural/functional characteristics and operational reactions, each of them studying life from a particular point of view, but still navigating among uncertainties.

However, although almost all of these branches agree that information is one of the important factors when we have to approach this important issue, no coherent and systematic achievements were obtained from the science of information point of view. Tarlaci raised on this line pertinent questions concerning the role of information at micro-physical (quantum) scale and macro-physical level [9, 10]. Starting from the philosophic concepts of information founded by Draganescu, showing that information plays a primordial role in matter structuration, with a highly increased role in living structures [11, 12] succinctly expressed by the relation: Structured matter + information $\Rightarrow$ Living structures, Gaiseanu has elaborated an Informational Model of Consciousness (IMC) [13] and an Informational Model of the Human Body (IMHB) [14], showing that information is a fundamental factor not only of the living systems operability in human, but also for the brain development on the evolutionary scale [14], by the gradual epigenetic adaptive integration of information into the living structure.

The Scrödinger's analysis shows that the living structures need a negentropy contribution to maintain their structure and functions, as a compensatory ordering process against the natural process of the entropy increasing in our material world, according to the second thermodynamic law [15]. That means the difference between the entropy in the final and initial state to be not positive, as required by the second thermodynamic law, but reversely. However, in his analysis Schrödinger did not taken into account the contribution of information to the structuring internal process of the living systems. On the basis of the new concepts of information, which allowed to describe successfully the informational circuits and systems of the human body, and of a comparative analysis of the living elementary unit - the cell, in this paper it is presented an Informational Model of the Living Structures (IMLS).

\section{Living / Non-living Info-matter Structuration and Specific Properties / Functions}

The deoxyribonucleic acid (DNA) is the main structural molecule of the living cell, containing all the necessary instructions assuring the functions and reproduction of the cell. The unit of life is the cell, from the simplest and singular one, able to function self-sufficiently/automatically and reproduce itself, i.e. the prokaryotic cell (archaea and bacterium without nucleus, internal membranes and organelles) and the more evolved version which is the eukaryotic cell (protozoa, fungi, plants and animals - where the nucleus/organelles are enclosed within a membrane (Figure 1 right side)), to the complex multicellular structures like plants, animals and human. To understand the specific properties of the living structures, it is necessary first of all to understand the structuration of living entities, comparatively with the non-living structures.

The basic material of a life structure is carbon, situated in the group IV of the universal table of the chemical elements like silicon - the material substrate for the preparation of the intelligent microprocessor structures of which our informational era fully benefit today. However, the silicon devices are intelligent/informational structures, but not alive. Such devices are prepared at high temperatures (around $1000^{\circ} \mathrm{C}$ ) to form junctions between oppositely (YES/NO) doped regions with donor-type (electrons) and acceptor-type ("holes" - positively charged) and electrical free charges [16]. Silicon atoms form predominantly crystalline solid structures, where the atoms occupy well-established sites in the crystalline lattice with minimum potential energy [17].

Carbon is more and more discovered in our informational era not only as a basic constituent of living structures, but also as a fascinating material serving as a graphene-type substrate/devices or connection nanotube-type devices [18]. In contrast with silicon, carbon can form large chains of covalently bonded molecules on the basis of associative/dissociative (YES/NO - Bit-type) reactions at the current temperature, with close activation energy in the range starting from 3.6-3.9 eV/bond for the $\mathrm{C}-\mathrm{C}$ covalence to $\sim 10$ $\mathrm{eV} /$ bond for $\mathrm{HC} \equiv \mathrm{CH}$ and $11.16 \mathrm{eV} /$ bond for $\mathrm{CO}$ [19]. Carbon can form therefore an extremely large number of compounds the organic components: there are close to ten million known carbon compounds, many thousands of which are vital to organic and life processes [20], especially with hydrogen, oxygen, nitrogen and phosphorus. In other words, the organic compounds are sensitive to change, so to information, on one side because of quite small differences in the chemical bond energies of the large variety of compounds belonging to this family and on the other side by the small modifications of the temperature or ambient conditions for which they are susceptible to change. The chemical reactions are informational processes, occurring by "embodiment/" "disembodiment" of information represented by loss (acceptor) or gain (donor) of information, because the state of the system (measurable by entropy) changes through such an interaction.

The composition of a bacterium, the simplest living organism, consists in 70\% water (an excellent solvent) and in $30 \%$ organic compounds, i.e. $15 \%$ proteins, $6 \%$ RNA (ribonucleic acid), $2 \%$ phospholipids, $2 \%$ polysaccharides, $1 \%$ DNA and 4\% small molecules [21]. The proteins represents a major part of organic compounds, and are formed by rests of amino acids with a basic composition of carbon, hydrogen, oxygen, and nitrogen. In human body there are necessaries 
only twenty amino acids to form proteins in cells, eleven fabricated by the human body itself, and nine obtained from foods, all of them participating in the genetic code (matter-related) information.

DNA, the main informational structure of the cell is situated in the nucleus of eukaryotic cell (Figure 1 right side) and is actually a warehouse of information, which shows an aperiodic configuration, in contrast with that of silicon, which forms specifically solid-state crystalline lattices. Just for this reason, DNA structure allows to accumulate a high quantity of information, due to the high (practically infinite) number of combinatory configurations between the four bases (nucleotides) binding the two very large helix-type strands of carbon chains. The bases are: Adenine (A), Thymine (T), Cytosine (C) and Guanine (G), but A may only bind the complementary $\mathrm{T}$ base and $\mathrm{C}$ only the complementary base $\mathrm{G}$, acting as YES/NO informational type operator pairs. The genetic patterns/mechanisms managed by the DNA molecule in the nucleic area, permit to decide the organism' development and structuration by the interaction with proteins. The main informational role of RNA is to read information stored in DNA and to transmit it by "messenger" (mRNA) agents, allowing to express/manifest this information during the cell replication [8]. The division of DNA molecule in two strands read by RNAs to replicate the cell is also a binary informational elemental process, as well as the gender differentiation and further reunification in the fertilized egg, patented by nature. In the cells of the nervous system in animals and human body, the transmission of information along the axon is performed by electrical YES/NO impulses [13], because the electrical pulse may pass (YES), only if the electrical potential at the entrance exceeds a minimal threshold value [22], so otherwise NO.

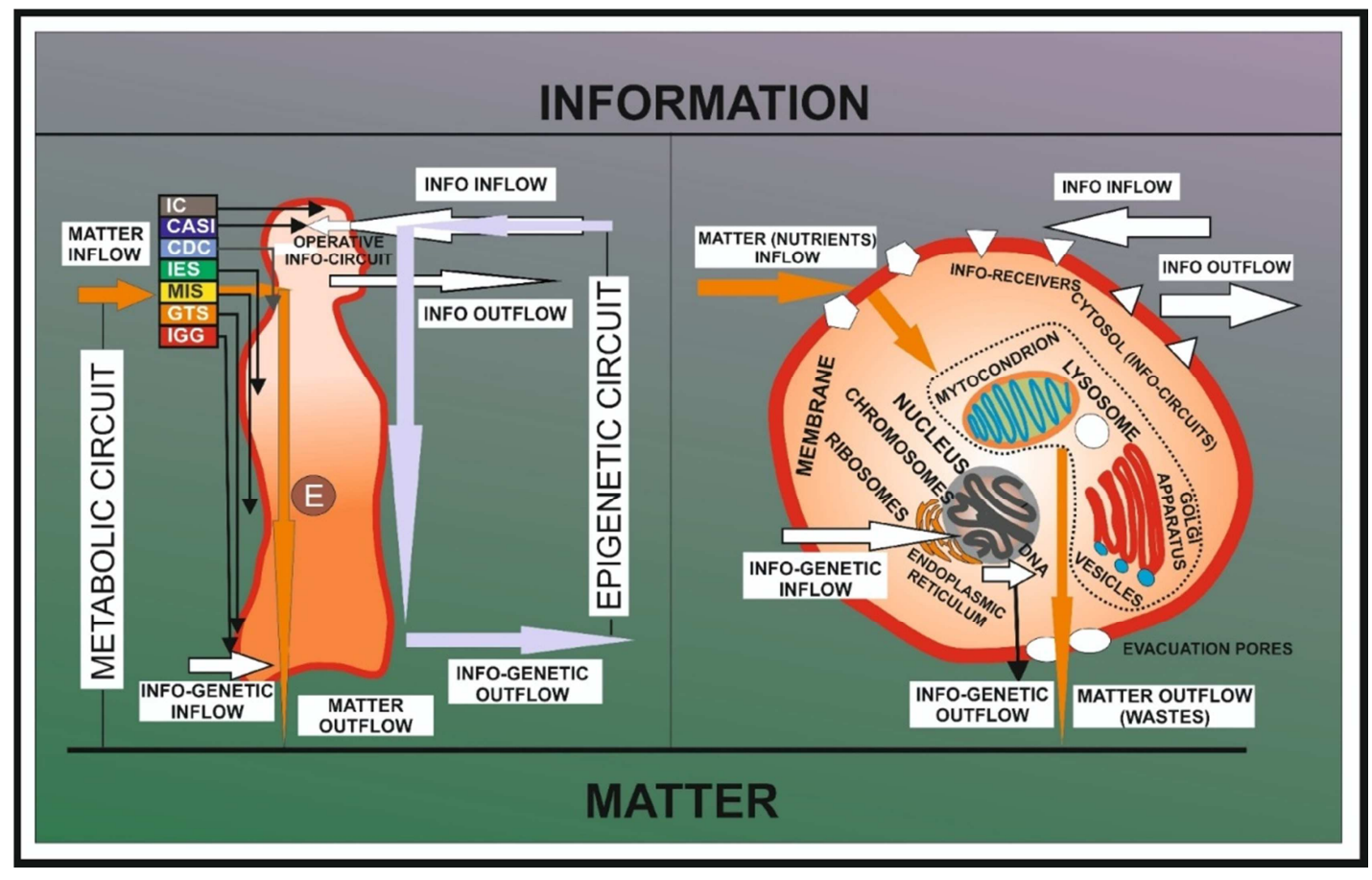

Figure 1. Schematic representation of the dynamics of matter and information circuits in human organism (left side) and in an animal eukaryotic cell (right side). In the left side of figure is shown also a schematic representation of the main informational systems of the human organism and their connection (black arrows) with the body organs/regions, according to the Informational Model of the Human Body (IMHB).

The energy in the cell is obtained by a quantum breakdown of chemical bonds of some organic components (carbohydrates, fats, proteins) in animals and by the photosynthesis quantum-assisted mechanism with absorption of photons in plants, which reacts with oxygen obtained from a "respiration" process in mitochondria organelles (Figure 1 right side), to release energy and excretory wastes products (carbon dioxide, uric acid, water and small molecules) [23]. The energy supplier is the adenosine triphosphate (ATP), synthetized from adenosine diphosphate (ADP) and inorganic phosphate, used for other biological processes. The ratio $\mathrm{C}(\mathrm{ATP}) / \mathrm{C}(\mathrm{ADP})$, where $\mathrm{C}$ represents the concentration, is an informational factor determining the cell to spread (YES/NO) information concerning the needs/function of the cell: if
$\mathrm{C}(\mathrm{ATP}) / \mathrm{C}(\mathrm{ADP})>1$, the cell can use ADP to work, if not, the balance initiates the ATP synthesis. Therefore, we have to observe that these processes in the mitochondria of the cell (Figure 1 right side) are precursors of the similar processes in the lung of animals. The energy achievement is a quantum assisted process, which takes place with release of free electrons and formation of electronic/protonic ( $\mathrm{H}+$ ions) gradients [8]. An additional process of energy achievement is started in cytosol (the water soluble cytoplasm) of the cell by an anaerobic process (fermentation) assisted by enzymes (which lower the barrier energy of molecule breakdown), finalizing also in mitochondria membranes.

The Endoplasmic Reticulum (ER), Golgi apparatus, lysosomes (Figure 1 right side) operate as organelles with 
specific digestive functions in the eukaryotic cells. The role of $\mathrm{ER}$ is to manage the lipid (fats) reactions and insulin production, operating actually as a precursor of the pancreas in the highly organized structures (animals). The transport of lipids and insulin, to name just these two representative proteins, is organized though an informational mechanisms, by means of specific amino acids as signaling orientation agents, which indicate the right address where the vesicles ("transporters" managed by Golgi apparatus) from fabrication place ER should go to lysosomes (acting as a spleen in the animal organisms), returning back toward the fabrication ER region. Such circuits and organelles operations according to rigorously-stablished informational rules and reactive pathways, are the precursors of the digestive/distribution systems in the multicellular organisms. In prokaryotic cells, where no organelles are present, the cytoplasm is the region where these processes take place.

The semitransparent membrane of the cell plays a fundamental role for the specific interchange of matter/energy/information with the environment, because it can permit (or not) by YES/NO selective mechanisms the transfer from/to the cell of the necessary nutrients and informational agents, according to the internal adaptation/survival necessities, protecting the specific conditions for the automatic processes inside of the cell "laboratory" and organelles, eliminating the wastes. The cell membranes are the corresponding precursors of the skin and coating tissues of the organs in animals.

The degree of disorder of the components of a system is evaluated from thermodynamic point of view by entropy, so the "negentropy" introduced by Schrödinger would reflect actually the decrease of the degree of disorder, signifying actually the increase of ordering in such a system. The living structures are therefore able to organize their configuration by "antientropic" [24]/"negentropic" mechanisms, absorbing actually information. The favorable change of entropy of a living structure is obtained basically by the metabolic processes, dedicated to the cyclical conversion of the absorbed matter/nutrients and energy (light in plants) from the external environment for internal energy achievement, necessary in the internal reconstruction/ordering (information gaining) work, eliminating wastes and heat (unconverted energy in work) in the environment. From the thermodynamic point of view, this process is similar with that operated by the refrigerator machine, working to throw outside the heat. The aggregation of small molecules to form proteins and the change of their configuration to folded $3 \mathrm{D}$ proteins is also a process with gain of information and loss of entropy [6]. Epigenetic processes not only represents the pathway patented by nature to absorb information from environment and to integrate it into the living structure [25], but also are an efficient and proactive way to lower their entropy and gain in organization/information on the evolutionary scale [26].

A discussion in terms of elementary thermodynamics applied to living (open) systems shows that a part from the work $\mathrm{W}$ consumed inside of the cell in metabolic processes from the internal energy $\mathrm{E}$ is "pumped" as a heat Qw in the environment, lowering the inner entropy in comparison with a non-living (static) system, where no internal work is produced, because the entropy of the dynamic (living) structure would be $[(\mathrm{Q}-\mathrm{Qw}) / \mathrm{Te}-\mathrm{Q} / \mathrm{Tc}]$, less than $(\mathrm{Q} / \mathrm{Te}-\mathrm{Q} / \mathrm{Tc})$ comparatively evaluated, where Te (final value) and Tc (initial value) would be the environmental and structure absolute temperature respectively. In classical thermodynamics however, the concept of information is not explicitly expressed, although movement/kinetics and information should be related concepts in the living systems [27], so a relation $\mathrm{dW}=\left(\mathrm{k}_{\mathrm{B}} \mathrm{T}\right)$ $\mathrm{dI}$ should be introduced to fulfill this goal [28], in which $\mathrm{dW}$ is the increment of work required in a reversible process at absolute temperature $\mathrm{T}$ in a system, and $\mathrm{dI}$ is the information obtained by this system measured as a reduction of entropy, $\mathrm{k}_{\mathrm{B}}$ the Boltzmann's constant. To produce 1 bit of information is necessary an energy cost of $\ln \left(2 \mathrm{k}_{\mathrm{B}} \mathrm{T}\right)=3 \times 10^{-21} \mathrm{~J}=18.5 \mathrm{meV}$, so more than 1 bit of information should be necessary to be released to have a significant effect at the current temperature [5]. To understand the level of such an informational intervention, we have to note that the order of magnitude of the quantity of information in prokaryotes cell, particularly in Escherichia coli bacterium is significant, of about $2 \times 10^{11}$ bits, confirmed by experimental studies [6].

Such an analysis in terms of information shows that the science of information becomes a necessary/imprescriptible tool not only to understand the living structures [29], but also to approach the design of the artificial mimic biosystems, with spectacular results in the realization of biocomputing devices with DNA or RNA, biotransistors, biomemories, image processors, image cryptosystems, neuronal genetic networks, neurostructures, biomedical structures compatible with the living structures, unimaginable with decades ago [18]. On this line of informational analysis, the Shannon's concepts on information [30], elaborated initially to describe/evaluate the information quantity in the (micro) electronic communication systems, are more general than Boltzmann thermodynamic theory, representing a more suitable quantitative basis for the evaluation of information in living systems [31].

Other specific strategies of the living structures like compartmentalization and semitransparent membranes, which act as specific "devices" for selective transfer of matter particles according to their size or chemical reactivity, are used to lower the entropy and gain in information. The osmotic process managed by semitransparent membranes of the cells [32], consisting in the diffusion of water to the more concentrated solute regions, even against the gravitation force in plants and animals is an example [6]. Specifically, the semitransparent cellular membrane allows also the selective transmission of information from and to the cell (Figure 1 right side), by specialized protein receptors incorporated into membrane, which may bind only complementary components to transmit information inside or outside of the cell by a YES/NO type informational mechanism. In the cells of the nervous system, the $\mathrm{Na}^{+} / \mathrm{K}^{+}$energetically-assisted pump effect against the concentration gradient near the cell membrane, allowing the formation of the electrical action potential and subsequent discharge by electrical input signals in axon, is 
also another example.

The complementarity of the organic compounds is one of the fundamental key explaining the specific behavior of the living structure operability in a binary YES/NO informational mode. On the basis of this dual YES/NO mechanism of complementary/ compensatory / contradictory driving forces or devices, it can be attained the equilibrium conditions and the stable health [3] at the small scale of the cell or at the body macroscale by homeostatic equilibrium. In the nervous system, this type of equilibrium is obtained by the activation of the excitatory/inhibitory neurotransmitters - assisted mechanisms in the specific vegetative sympathetic/parasympathetic nervous systems.

Compartmentalization consists in the partition of the cell/living structure available volume in smaller regions, where the specific life reactions may operate. The organelles in eukaryotic cells and organs in the human and subhuman organisms are eloquent examples of compartmentalization. One of the most advantage of compartmentalization is the increasing of the cells ordering structuration, so the lowering of their entropy by absorption of information [33, 6], as a natural evolutionary process from simple to more complex structuration [7], inspiring the design for the realization of artificial cells which mimic the natural biologic systems [34].

\section{Info-communication and Informational Model of the Living Structures}

The imprescriptible principles of the cell life discovered in biology [35] could be discussed from informational perspective as follows: (1) cells are always in motion (so acts as informational systems); (2) cells should be permanently in contact with their environment (as a source of food, energy and information for adaptation); (3) DNA integrity and survival is of the high priority (as informational manager of cell); (4) DNA allows the info-stored transmission by RNAs and proteins (info-transmitter agents assuring the fundamental functions of the cell); (5) the internal and external membranes assure the selective operability of macromolecules (breakdown of their bonds and energy production, selective info-communication, partition in organelles for an efficient functionality, prefiguring the specialized organs in the superior multicellular organisms); (6) chemical bonds and ion gradients serve as cellular fuel (obtained by quantum mechanic/informational mechanisms, as described above); (7) signaling inter-communication networks and pathway reactive mechanisms are the nervous system of a cell (attributes and circuits of cell internal/external info-communication); (9) the cell division is the most vulnerable cycle of cell (info-managing process of the cell partition and reproduction).

The specific functions of human and subhuman organisms are organized in characteristic (compartmentalized) informational regions of the brain, structured by an info-evolution process [26] to increase the efficiency/complexity of info-operability, as discussed above.
The Informational System of the Human Body (ISHB) [13] consists in: (1) the Center of Acquisition and Storing of information (CASI) [36], managing the sensory and memory system; (2) the Center of Decision and Command (CDC) [2], making decisions and transmitting the commands to the motor execution elements of the body; (3) the Info-Emotional System (IES), responsible for the emotions and emo-states [37]; (4) the Maintenance Informational System (MIS), managing the automatic processes of absorption/desorption (digestion) [14]; (5) the Genetic Transmission System (GTS), administrating the genetic creation/transmission [38]; (6) the Info-Genetic Generator (IGG), managing the body development during the lifespan [39]; (7) the Info-Connection is a special center responsible for the extracorporeal and special extra-sensitivity phenomena related with Near-Death Experiences (NDEs) [40], Mystic and Religious Experiences (RMEs) [41] and with the so called paranormal phenomena [42]. These centers are neuro-connected with specific regions of the brain [38], assuming the role of coordination and are represented schematically in the left side of the Figure 1.

The Informational Model of the Human Body (IMHB) takes into account the matter-related metabolic circuit and the internal organs acting as info-transducers/execution elements [14]. The human body is therefore connected to information and to matter poles of the organism (as can be seen from Figure 1), operating as a bipolar info-matter "device". The input information received in IC/CASI is operated in CDC for adaptive decision and transmission to the info-transducers (muscles) as an informational output (specifically expressed by verbal attitude), IES reacts to information and this reaction is felt as emotions - with participative contribution to decisions, MIS assures the stable maintenance of body by the management of the metabolic circuit (foods/air/water absorption/desorption processes), while GTS acts as a genetic informational output and IGG as a genetic informational input of the organism. Three main circuits are to be distinguished from Figure 1: metabolic (body maintenance) circuit managed by MIS, characteristic for the matter/energy processing, Operative Informational circuit/System for adaptation (OIS mainly composed by CASI+CDC+IES), and epigenetic circuit, allowing the integration of input information into the genetic system as a consequence of a long-time repetitive or intensively felt signal/cue [43]. We have to note again that from the organizational/primness information-gain perspective, the distinctive functions described by IMHB appears as a natural consequence of the brain compartmentalization in specialized functional regions on the evolutionary scale [26], acting in a modular way [43], which allows a better operational efficiency, according with the adaptive necessities.

In the light of the above analysis/discussion and after the examination of the main functions of a cell, either prokaryotic (bacterium) or eukaryotic (animal/plant) cell, we can discover that such functions are similar with that defined by IMHB (Figure 1, right side). The equivalent IGG from the (multicellular) human organism is represented in cell by nucleus/DNA-RNA operators, assuring the genetic 
transmission to the new generation. The inter-generational genetic transfer of information in cell (GTS $\rightarrow$ IGG) flows through DNA chromosomes chemical mechanisms: replication during reproduction/transcription of DNA information in RNA messenger (mRNA) as follows: DNA $\rightarrow$ mRNA $\rightarrow$ polypeptide $\rightarrow$ protein [6]. MIS is represented in cell by the automatic/driving chain reaction pathways which assure the digestion of the primary nutrient components, oxygen and water, within the metabolic inflow/outflow circuit including membranes, Golgi apparatus, lysosomes, vesicles, mitochondria, as described above and represented in Figure 1 as a region defined by the dashed line. The cell communication with the environment is assured by means of the receptors of the semitransparent membrane, allowing to bind selectively only the informational chemical agents complementarily structured. The informational inflow from the external membrane is then initiated in cytosol by means of specific cascade of chemical reactions, depending on the nature of the input signal $[5,44,45]$. The reactive response of the cell consists in two main components: (i) one of them represented by an external outflow of information to the next cell or to the external environment, which is the "decisional attitude", corresponding to CASI (sensorial reception) + CDC (specific operational selective process of response); (ii) another reactive component is sensitively "felt" by the cell itself in its elemental "sentient" IES system, as a corresponding repercussive reactivity inside of each component of an interacting system [41], preserved internally as an "emotional" memory. Biological memory, heritable or not, can be defined as a stable-standardized cellular response to a transient signal. One of the ways to comply with this task is through transcriptional response assisted by molecules, regulating gene expression [45]. Memory is a fundamental info-accumulation of experiential events maintained in a stand-by state, imprescriptible for learning and adaptation of the living systems, serving as reference for further decisions. CASI in a cell is represented by the system of notifications/perceptions/memorization of the informational signals through chemical components.

In opposition with the human, it is more difficult to understand how an emotional system would work in subhuman or inferior living structures. Some recent studies shows however that like in human, the primitive "emotions" should be also perceived in the primitive living structures as a binary positive/negative, "good/bad" (YES/NO) experiential/selectively learned "lessons", classified according to previous memorized experience [18], playing a regulatory role as an adaptive process in E-coli bacterium [46] and the successive organisms on the evolutionary scale [26]. A generalization of IES in a living system, redefined as an Informational Reactive-Sentient System (IRSS), can be described therefore as a sentience interaction processes [41], supported by the internal molecular self-regulatory balance, when a stimulating/proactive informational factor (specifically the input information) triggers/determines an interaction with another component of the system, this one transmitting the signal to a sensitive specialized part of such a system, to be interpreted as an "emotion". Not only the multicellular organisms, but also individual cells are able to detect therefore the environmental conditions, to read them and to react consequently in a sensitive way, memorizing their life experience as comparative reference for further adaptation. The cell accomplishes therefore with cognitive and sentient tasks and as additional evidences, it should be noted that the gametes of the sperm and oocytes use some sorts of neurotransmitters for communication, and even plant cells dispose of integrated sensorial/sentient networks for the detection and interpretation of the environment conditions [47].

It seems also more difficult to assign a significant role to IC in inferior living systems. However, analyzing empirical observational data on such systems, it can be revealed also the important role of IC even in the primitive unicellular prokaryotic organisms like bacteria, which are able to communicate synchronously with other members, in order to form geometrically organized colonies [5], or at birds oriented though extra-sensorial signals assisted by quantum processes for migration, and at some animals and fishes sensing in advance the coming natural cataclysms or bad weather [48], so IC activity can be assimilated with extra-sensitive communication.

The epigenetic circuit represented in the left side of Figure 1 is dedicated to the integration of information for long-term adaptation, inter-generationally transmitted at human [25]. However, because this is actually an intimate cellular mechanism [13], it is evident that such a mechanism is also operational in primitive living structures, explaining the evolutionary process by gradual adaptation to the change of the environmental conditions. Therefore, the cell is actually a bipolar info-matter organism, like the human system, connected to information and matter by the informational circuit and metabolic (matter-related) circuit sustaining the cell body structure, which absorbs information from environment and can integrate it by the epigenetic mechanisms/circuit in the genetic code of the cell. Such a process starts by the connection of CASI to the same type of long-time informational inflow imposed by the external conditions (Figure 1 left side), ending in GTS (epigenetic traits acquirement), via CDC (decision), IRSS (primitive reactive "emotions", co-participating to the decision), MIS (automatic info-abilities acquirement), within an hierarchical organized strategy for survival and adaptation. In the particular case of human, the vision and olfactory sensing receptors in CASI operate by means of quantum-assisted mechanisms, pointing out the participative role of quantum-assisted processes in living structures at any scale level.

The inflow and outflow of information in the inter-communication process in cell represented in the right side of Figure 1 is created by the info-operational agents consisting mainly in amino acids and derivatives, proteins and peptides, and cells can react by a rapid, transitory or slow/long-term response. The receptors operate selectively, so there are practically two main ways by which the target cells 
can process the in-coming signals: YES (initiation) or NO (inhibitory) way, as an informational binary operational (Bit) unit. Paracrine-type signaling is referred only to the surrounding cells and not enter in the blood vessel circuits of the animals, while the endocrine signaling (hormones and other molecules) enter in the blood stream for the distance communication. The cellular reaction and combinations of signaling molecules generates different types of responses, fundamental for the cell operability: survival, growth and division, differentiation or death. The intra-cellular transmission of information involves multiple steps and proteins in biochemical reaction pathways, each of them determining a specific response/operation like an informational device, familiarly also in the operational microelectronic circuits and systems: transduction, relaying, amplification, integration, spreading, modulation, activation. It is to be noted also that negative or positive feedback loops could regulate the strength/frequency of signals, like in the specialized informational microelectronic devices.

The intracellular communication is also based by a binary (informational) YES/NO process of reception/transmission/transduction of the external signals by "cognate"/recognition complementary binding proteins in membrane and receptors in cytosol, or in nucleus [49]. A switching YES/NO fundamental role is also played by enzymes, which can activate/deactivate various internal processes like the production of messengers or signal amplification, and can become active in a timely and locally way. It is to be noted also that the informational carrier agents could play the role of signal, receptor, adapter, effector, adapting the operational process inside of the elementary cell, as can be observed in the behavior of operational processes at macro level scale in the human body described in IMHB [14]. On the line of the reproduction of the functions of the living intelligence, the specific nerves communication serves now as a natural model for the construction of artificial memristor mimic devices in neuromorphic systems [50], biocomputing systems (with DNA/RNA) and biosystems [18].

According to the above discussion, the cell is therefore a dynamic multi-processing device functioning in a steady-state mode, able to adapt itself depending on the signals received from outside. The modular cell construction allows the signaling proteins to interact with multiple upstream and downstream currents in an informationally controlled mode, the proteins playing a multilateral/multivalent and flexible role in the info-transmission of input and output signals, as it is characteristic for the human brain operability of the automatic Programmed Informational System (PIS = MIS + GTS + IGG + IC) [18]. One outcome from the above analysis shows that the main functional mechanisms in the living structures are based on YES/NO compensatory/complementary type of processes like excitatory/inhibitory, excess/lack, activation/deactivation, overpressure/sub-pressure, allowing to maintain the active existence of the living structures, assuring not only a dynamic steady-state equilibrium/health, but also that small deviations of such a balance equilibrium produce automatic triggered mechanisms/events activating the necessary driving forces for the re-establishment of the defining (homeostatic) equilibrium and health.

A microelectronic device, suitably prepared to operate with informational flows, can be found in two main situations: (i) in a stand-by regime, without external signals, but polarized with a suitable biasing voltage to assure a steady-state condition, and (ii) under operational mode, when a signal is applied to an input connection to initiate a processed signal response in the output terminal. A similar situation is observed from the analysis of the cell operability: the steady-state conditions are fulfilled by the metabolic circuit, assuring the maintenance of the stand-by cell structure and its "polarization" (energetic resources for matter and information in/out-flows), and the informational modulator inflow is operated by OIS processes, to obtain an informational outflow as a reactive response. However, in contrast with a non-living microelectronic device, the cell units can reconfigure their informational structure by info-integration/learning epigenetic processes. A living structure can be defined therefore as a dynamic steady-state equilibrium self-organized system, modulated by a received information, which induces small deviations from the main equilibrium axis according to the tolerance limits, and responding/reacting at the external stimulus by permanent communication with the environment and own components to assure the change of matter ("polarization") and information for adaptive and survival processes, on the basis of homeostatic YES/NO compensatory mechanisms. Regarded from this perspective, the living structures behave/operate like an informational device.

The above analysis and discussion allow the foundation of the Informational Model of the Living Structures (IMLS), describing them as informational bipolar info-matter devices, from elemental units to the multicellular complex structures, able to maintain themselves the stand-by dynamic equilibrium state to preserve their integrity and functionality on the basis of the metabolic matter-related circuit, and to operate by means of the Informational System of the Living Structure (ISLS), which can be described in a similar way with ISHB by means of seven main functional components as follows:

$\mathrm{ISLS}=[\mathrm{IGG}+\mathrm{GTS}+\mathrm{MIS}+\mathrm{IRSS}+\mathrm{CDC}+\mathrm{CASI}+\mathrm{IC}]_{\mathrm{ISLAS}}(1)$

where the activity of each component is interpreted in terms of information as described above. Therefore, similarly with the PIS (Informational Programmed System) defined in IMHB, the Programmed Informational System of the Living Structures (PISLS) including cells, can be defined as:

$$
\text { PISLS }=[\mathrm{MIS}+\mathrm{GTS}+\mathrm{IGG}+\mathrm{IC}]_{\mathrm{ISLS}}
$$

where the role of GTS and IGG is evidently complied by DNA/RNA components of nucleus and the associated agents, while the info-selection role of IC by info-YES/NO suitable selection switching mechanisms involved in extra-communications.

The adaptive reactive response of the living structures (including cells), could be also defined similarly with the 
Operative Informational System (OIS) in ISHB, sustained by the Operative Informational System of the Living Structures (OISLS), defined as follows:

$$
\text { OISLS }=[\mathrm{CASI}+\mathrm{CDC}+\mathrm{IRSS}]_{\mathrm{ISLS}}
$$

which can finalize with the intervention even in the core of the informational system, which is the DNA structure, by epigenetic mechanisms, without modification of the genetic main structure, by means of the operability of PISLS. Such mechanism explains the long-term adaptation and evolution, as discussed above.

The equations (1) - (3) represent a generalization of the ISHB applicable for the living structures, described in terms of information and matter, defined through (i) PISLS, composed by automatically driven processes strictly/rigorously info-managed on physical-chemical pathways to maintains the stabilized (homeostatic equilibrium), and through (ii) OISLS, an info-operational system able to memorize, decide and transmit a reactive sentient response to the environment and/or to itself structure for adaptation. This generalized informational model agrees/supports the Draganescu's philosophic concepts on information earlier discussed [12, 27], allowing an advanced understanding of the living operability from the informational point of view and clarification of the local operability/support mechanisms/processes in the living structures, still not understood up to date. Moreover, such an analysis and modeling open new perspectives for the design and application in artificial biosystems, mimicking the functions and operability of the natural living structures.

The IMLS shows that these structures are able to provide themselves the necessary energy, acting as functional devices not only to maintain their ordering structure against the disordering tendency dictated by the second thermodynamic law, but also to modify their functionality and even their structure for adaptation to the external stimuli and cues, and evolve through acquirement of new traits encoded by means of epigenetic mechanisms. The living structures operate like a self - "polarized" informational device by means of the metabolic circuit, and react/respond to the external/internal stimuli, which modulate their functionality within the tolerance limits, by a reaction signal ("attitude") to communicate with themselves and/or environment and their similar living units/entities. The living structures are able on this way to organize their configuration and operability as a function of the internal or environmental conditions, which they are continuously dependent of, to adjust them for short (attitude) and long-term (epigenetic) adaptation/survival. The time-limited life cycle is a consequence of the YES/NO confrontation between the (external) entropic (NO) and (internal) antientropic (YES) driving forces "fighting" for supremacy. The patented life solution to such a confrontation was to reproduce the self entity by the creation of the new generation of living structures, assuring the species survival. Shortly, in terms of informational devices, OISLS modulates with input external signals the circuits of PISLS, which assures the "stand-by" operability/maintenance of the living device, to return an output reactive informational response, which can induce epigenetic restructuring consequences in PISLS.

\section{Conclusions}

Stating as a reference the Schrödinger's basic question "what is life" and Draganescu's philosophic concepts on living, there were analyzed in terms of information the structures and functions of the living systems, from the cell unit to the human organism, concluding first of all that the living systems are structured on the carbon "king" chemical element, which exhibits an extremely high ability to form a large range of associative/dissociative complementary bonding compounds of the type YES/NO informational (Bit) units under small variations of reaction conditions. The negentropy invoked by Scrödinger to explain the self-organizing activity of the living systems is actually a consequence of the informational structuring, and is obtained mainly by compartmentalization (on the self-organizing evolutionary scale), chemical reactions to aggregate/organize small molecules in folded big macro-molecules, energy generation and conversion in internal work for metabolic processes and especially by the elimination of heat, the absorption of information to convert it in structural properties within the epigenetic processes for adaptation.

The info-components and informational processes in cell, as a smallest self-organized and self-supporting own living structure, were discussed referring to DNA informational structure and to organelle components of the eukaryote animal cells, showing typical functions with that of multicellular organs. The dynamics and communication of the living systems were discussed in terms of information, showing that they operate on reactive functional pathways for transduction, relaying, amplification, integration, spreading, modulation, activation, like in the microelectronic/microsystems circuits and systems. Similar functions like the informational components in human body described by ISHB were identified also in the elementary living cell. From such an analysis it was deduced that the equilibrium of organism is based on contrary/complementary/compensatory YES/NO processes like excitatory/inhibitory, excess/lack, activation/deactivation, overpressure/sub-pressure, allowing to maintain its active/dynamic existence. A living structure is therefore basically a dynamic steady-state equilibrium self-organized system, maintaining itself by matter inter-change with environment and modulated by received information, which induces small deviations from the equilibrium state, operating in a similar mode like the informational microelectronic devices. It was therefore deduced that the living organisms, independently on the scale size, operate by three main streaming circuits assuring the living functions: (1) the metabolic matter-related circuit, (2) the operative informational circuit for adaptation, and (3) the evolution epigenetic circuit for the gradual integration of information in the central informational structure - DNA.

It was founded and described the Informational Model of the Living Structures (IMLS), and the Informational System 
of the Living Structures (ISLS), with similar functions on the entire scale size, from unitary to multicellular living structures, which is composed by seven informational centers, namely [CASI, CDC, IRSS, MIS, GTS, IGG, IC] $]_{\text {ISLS, and were }}$ identified the specific functions of each of them. It was deduced in this way that the living structures operate like a self - "polarized" bipolar info-matter informational device and react/respond to the external informational stimuli, which modulate their functionality within the tolerance limits.

\section{Acknowledgements}

To my son Adrian Gaiseanu, eminent specialist, son and father, and to his family.

To my daughter Ana-Maria Gaiseanu, highly following and showing the best qualities of life in family, profession and society.

Special dedication to Acad. Florin Filip and to the members of the Science and Technology of Information in the Romanian Academy.

In memoriam of Academician Mihai Draganescu, the founder of Science and Technology of Information.

In the memory of my loved parents, Distinguished (Magna cum Laude) Professors Emanoil and (Emeritus) Florica Gaiseanu, of my brother Professor Constantin Gaiseanu and of all family members who were part of my Life.

\section{References}

[1] Gaiseanu F. (2019). Language Patterns and Cognitive-Sentient Reality: Certainty/Uncertainty in Cognitive-Sentient Exploration of Reality, Chapter in Media Models to Foster Collective Human Coherence in the PSYCHecology, Ed. Stephen Brock Schafer, USA, IGI Global: 49-72. DOI: 10.4018/978-1-5225-9065-1.ch003.

https://www.igi-global.com/gateway/chapter/229328

[2] Gaiseanu, F. (2018). Destiny or free will decision? A life overview from the perspective of an informational modeling of consciousness Part II: attitude and decision criteria, free will and destiny. Gerontology \& Geriatric Studies 4 (1): 354-360. https://crimsonpublishers.com/ggs/pdf/GGS.000576.pdf.

[3] Gaiseanu, F. (2020). Informationally-assisted equilibrium and health: specific ACC contribution from the perspective of the informational model of consciousness. EC Psychology and $\begin{array}{llll}\text { Psychiatry J., } & 9 & \end{array}$ (https://www.ecronicon.com/ecpp/ECPP-09-00692.php) $37-49$.

[4] Gaiseanu, F. (2018). Near-death experiences and immortality from the perspective of an informational modeling of consciousness. Gerontology \& Geriatric Studies, 2 (3): 1-4 https://crimsonpublishers.com/ggs/pdf/GGS.000538.pdf.

[5] Jacob EB, Shapira Y, Tauberd AI. (2006). Seeking the foundations of cognition in bacteria: From Schrôdinger's negative entropy to latent information, Physica A, 359: 495-524.

[6] Davies CW, Rieper E, and Tuszynski JA. (2013). Self-organization and entropy reduction in a living cell,

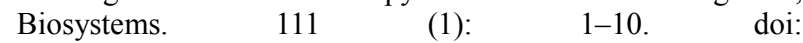
10.1016/j.biosystems.2012.10.005.
[7] Velazquez JLP. (2009). Finding simplicity in complexity: general principles of biological and nonbiological organization, J Biol Phys, 35: 209-221. DOI: 10.1007/s10867-009-9146-z.

[8] Adams JU, Ph. D. (2010). Essential of Cell Biology, Edited by Clare O'Connor\& Adams JU, Ph. D, Scitable, Cambridge, MA: NPG Education: 1-100.

[9] Tarlaci S. (2011). Quantum Physics in Living Matter: From Quantum Biology to Quantum Neurobiology, NeuroQuantology, 9 (4): 692-701.

[10] Tarlaci S., and Pregnolato M. (2016). Quantum neurophysics: From non-living matter to quantum neurobiology and psychopathology. International Journal of Psychophysiology, 103: 161-73. doi: 10.1016/j.ijpsycho.2015.02.016.

[11] Draganescu, M. (1990). Information of matter. Bucharest, Ed. Romanian Academy. (Informatia materiei (in Romanian). Bucuresti: Editura Academiei Române.

[12] Draganescu, M. (1979). The depth of the material world. Bucharest: Ed. Politica. (Profunzimile lumii materiale (in Romanian). Bucuresti: Editura Politica.

[13] Gaiseanu F. The Informational Model of Consciousness: Mechanisms of Embodiment/Disembodiment of Information, NeuroQuantology, $17 \quad$ (4), $\quad 1-17, \quad 2019 \mathrm{c}$. https://www.neuroquantology.com/article.php?id=1322.

[14] Gaiseanu F. Information-Matter Bipolarity of the Human Organism and Its Fundamental Circuits: From Philosophy to Physics/Neurosciences-Based Modeling, Philosophy Study 2020b; 10 (2): 107-118. doi: 10.17265/2159-5313/2020.02.002. http://www.davidpublisher.com/Public/uploads/Contribute/5e5 b3d8e74433.pdf.

[15] Schrödinger E. What is Life? The Physical Aspect of the Living Cell, Cambridge: Cambridge University Press 1944.

[16] Gaiseanu F. (2013). Contributions to the Modelling and Simulation of the Atomic Transport Processes in Silicon and Polysilicon and Applications. PROCEEDINGS OF THE ROMANIAN ACADEMY, Series A, 4 (4): 376-384; www.acad.ro/sectii2002/proceedings/doc2013-4/15-Gaiseanu.pdf.

[17] Gaiseanu F. (2017). Modeling and Simulation of the Impurity Diffusion and Related Phenomena in Silicon and Polysilicon Systems in Microfabrication and Micromachining Technologies. Annals of the Academy of Romanian Scientists, Series on Science and Technology of Information, 10 (1): 41-78. http://aos.ro/wp-content/anale/IFVol10Nr1 Art.4.pdf.

[18] Gaiseanu, F. (2020). Physics of consciousness and life: informational model of consciousness - information in neurosciences, biocomputers and biosystems; (Fizica constiintei si a vietii: modelul informational al constiintei informatia in neurostiinte, biocomputere si biosisteme (in Romanian), GlobeEdit (OmniScriptum International Group, Germany).

https://www.amazon.com/Fizica-Conștiinței-Vieții-Informațio nal-Neuroștiințe/dp/6139421705.

[19] Anonymous. (2020) Wikipedia, https://en.m.wikipedia.org/wiki/Bond-dissociation_energy

[20] Anonymous. (2003-2008). Carbon. Los Alamos National Laboratory. Chemistry Operations 2003-2008 https://web.archive.org/web/20080913063402/http://periodic.1 anl.gov/elements/6.html. 
[21] Alberts B., Johnson A., Lewis L., Morgan D., Raff M., Roberts K., Walter P. (2015). Essential Biology, 6-th edition; Published by Garland Science, Taylor \& Francis Group.

[22] Zhang J. (2019). Secrets of the Brain: An Introduction to the Brain: Anatomical Structure and Biological Function, arXiv: 1906.03314v1 [q-bio.NC]: 1-34.

[23] Garbi L., and Larsen PS. (2008). Bioenergetics: Its Thermodynamic Foundations. Cambridge University Press.

[24] Bailly F., Longo G. (2009). Biological Organization and Antientropy, Journal Biological Systems, 17 (1): 63-96.

[25] Gaiseanu, F. (2019). Epigenetic information-body interaction and information-assisted evolution from the perspective of the informational model of consciousness. Archives in Biomedical Engineering \& Biotechnology 2 (2): 1-6. DOI: 10.33552/ABEB.2019.02.000532

https://irispublishers.com/abeb/pdf/ABEB.MS.ID.000532.pdf

[26] Gaiseanu, F. (2020). Information based hierarchical brain organization/evolution from the perspective of the informational model of consciousness. Archives in Neurology \& Neuroscience 7 (5): 1-9. ANN.MS.ID.000672. DOI: 10.33552/ANN.2020.07.000672.

https://www.academia.edu/42766159/Information_Based_Hie rarchical_Brain_Organization_Evolution_from_the_Perspecti ve of the Informational Model_of Consciousness.

[27] Gaiseanu F. (2016). Consciousness as Informational System of the Human Body. Consciousness and Life Physics, Cosmology and Astrophysics Journal, 16 (1): 14-25. http://physics.socionic.info/index.php/physics/article/view/227 $/ 182$

[28] Smith E. (2008). Thermodynamics of natural selection I: Energy flow and the limits on organization. Journal of Theoretical Biology, 252: 185-197. and II: Chemical Carnot cycles, Journal of Theoretical Biology Evolution, 52: 198-212.

[29] Gaiseanu F. (2019). Informational Model of Consciousness: From Philosophic Concepts to an Information Science of Consciousness, Philosophy Study 9 (4): 181-196. http://www.davidpublisher.org/Public/uploads/Contribute/5d1 c009c3567e.pdf DOI: 10.17265/2159-5313/2019.04.002.

[30] Shannon CE. (1948). A mathematical theory of communication. Bell Syst. Tech. J., 27 (379-423): 623-656.

[31] Ben-Naim A. (2017). Entropy, Shannon's Measure of Information and Boltzmann's H-Theorem, Entropy, 19: 48; 1-18. doi: 10.3390/e19020048.

[32] Cherak S.J., Gugala N., and Turner RJ. (2017). Membrane Transport, Basic Chemistry, Austin Publishing, 1-33, Basic Biochemistry. www.austinpublishinggroup.com/ebooks.

[33] Marín D., Martín M., Sabater B. (2009). Entropy decrease associated to solute compartmentalization in the cell, BioSystems, 98: 31-36.

[34] Chen A., and Silver P. (2012). Designing biological compartmentalization, Trends in Cell Biology, 22 (12): 662-670.

[35] Plopper G. (2016). Principles of the Cell Biology, 2-nd Edition, Jones \& Bartlett Learning.

[36] Gaiseanu F. (2019). Destiny or Free Will Decision? A Life Overview From the Perspective of an Informational Modeling of Consciousness Part I: Information, Consciousness and Life Cycle. Gerontology \& Geriatric Studies, 4 (1): 400-405. https://crimsonpublishers.com/ggs/pdf/GGS.000586.pdf

[37] Gaiseanu F. (2018). Information: from Philosophic to Physics Concepts for Informational Modeling of Consciousness, $\begin{array}{llll}\text { Philosophy Study, } 8 & \text { (8): } 368-382 . & \text { doi: }\end{array}$ 10.17265/2159-5313/2018.08.004.

http://www.davidpublisher.org/Public/uploads/Contribute/5c0 6323653cd2.pdf.

[38] Gaiseanu F. (2019). Informational Neuro-Connections of the Brain with the Body Supporting the Informational Model of Consciousness, Archives in Neurology \& Neuroscience 4 (1): 1-6. ANN. MS. ID. 000576. DOI: 10.33552/ANN.2019.04.000576.

https://irispublishers.com/ann/pdf/ANN.MS.ID.000576.pdf.

[39] Gaiseanu F. (2019). The Silent Voice of Those Who are no Longer: Transgenerational Transmission of Information from the Perspective of the Informational Model of Consciousness. Gerontology \& Geriatric Studies, 5 (1): 482-488. DOI: 10.31031/GGS.2019.05.000604.https://crimsonpublishers.com /ggs/pdf/GGS.000604.pdf.

[40] Gaiseanu F. (2017). Quantum-Assisted Process of Disembody Under Near-Death Conditions: An Informational-Field Support Model. NeuroQuantology, $15 \quad$ (1): 4-9. https://www.neuroquantology.com/article.php?id=1645.

[41] Gaiseanu F. (2019). Human/Humanity, Consciousness and Universe: Informational Relation, NeuroQuantology, 17 (5): 60-70. https://www.neuroquantology.com/article.php?id=1232

[42] Gaiseanu F. (2017). An Information Based Model of Consciousness Fully Explaining the Mind Normal/Paranormal Properties. NeuroQuantology, 15.2: 132-140. https://www.neuroquantology.com/article.php?id=1676

[43] Gaiseanu F. (2019). Informational Mode of the Brain Operation and Consciousness as an Informational Related System, Archives in Biomedical Engineering \& Biotechnology, 1 (5): 1-7. ABEB.MS.ID.000525. https://irispublishers.com/abeb/pdf/ABEB.MS.ID.000525.pdf

[44] Burrill D. R., and Silver P. A. (2010). Making cellular memories, Cell, 140 (1): 13-18. doi: 10.1016/j.cell.2009.12.034.

[45] Mathisa R., and Ackermann M. (2016). Response of single bacterial cells to stress gives rise to complex history dependence at the population level, PNAS Early Edition, 1-6, www.pnas.org/cgi/doi/10.1073/pnas.1511509113.

[46] Peil K. T. (2014). Emotion: The Self-regulatory Sense, Global Adv Health Med., 3 (2): 80-108. DOI: 10.7453/gahmj.2013.058.

[47] Shanta B. N. (2015). Life and consciousness - The Vedāntic view, Communicative \& Integrative Biology, 8: 5, e1085138 (1-12), DOI: 10.1080/19420889.2015.1085138.

[48] Marais A., Adams B., Ringsmuth A., Ferretti M., Gruber M., Hendrik R., Schuld M., Smith S., Sinayskiy I., Krūger T., Petruccione F., Grondelle R. (2018). The future of quantum biology, J. R. Soc. Interface, 15: 20180640: 1-15.

[49] Krauss G. (2014). Basics of Cell Signaling, in Biochemistry of Signal Transduction and Regulation. 5th Edition. WILEY-VCH Verlag GmbH \& Co. KGaA, Weinheim ISBN: 978-3-527-31397-6. 
[50] Indiveri, G., Linares-Barranco B., Legenstein, R., Deligeorgis G., and Prodromakis, T. (2013). Integration of nanoscale memristor synapses in neuromorphic computing architectures,
Nanotechnology
24
384010
$(1-13)$
doi:

$10.1088 / 0957-4484 / 24 / 38 / 384010$. 